\title{
A tattoo pigmented node and breast cancer
}

\author{
Soran, A, Kanbour-Shakir A, Bas O, Bonaventura M \\ Magee-Womens Hospital of UPMC, Pittsburgh, PA, USA. asoran@magee.edu
}

\begin{abstract}
Over the last decade, the axillary SLNB has replaced routine ALND for clinical staging in early breast cancer. Studies describe a potential pitfall in the identification of a true sentinel node during surgery due to lymph node pigmentation secondary to migration of tattoo dye. These pigmented "pseudo-sentinel" nodes, if located superficially in the axilla, may mimic the blue sentinel node on visual inspection, therefore missing the true sentinel node and potentially understaging the patient. Here, we present a case report of a breast cancer patient with a tattoo and discuss the importance of tattoo pigment in the LN (Fig. 1, Ref. 8). Text in PDF www.elis.sk. Key words: breast cancer, sentinel node, tattoo, upgrade.
\end{abstract}

Abbreviations: SLNB - sentinel lymph node biopsy, ALND axillary lymph node dissection, LN - lymph node, SLN - sentinel lymph node.

In recent years, tattooing has dramatically increased in popularity among both men and women. Tattooing is no longer confined to sailors, gang members, prisoners and specific subcultures, but includes career women, college students and celebrities. Statistical data shows that $14 \%$ of Americans (all ages), $36 \%$ of adults $18-25$ years and $40 \%$ of adults $26-40$ years have at least one tattoo while the practice is gaining social acceptability and is becoming more popular. The preferred tattoo regions, in order, are arms, trunk, legs, feet, head/neck, hands, trunk/arms and genital area (1-2-3). The process of tattooing, which involves the repetitive piercing of the skin with ink-filled needles, results in a minor skin injury and permanent imprint of a design. Histologically, it creates a typical acute inflammatory reaction immediately, and within a few days, macrophages engulf the pigment (pigment-laden macrophages). It is well known that tattoo pigment can migrate to the regional lymph nodes and cause varying degrees of inflammatory reaction. Lymphadenopathy can result when tattoo pigment drains to regional lymph nodes and causes hyperplasia. Studies in mice demonstrate that by day 3 , pigment is already detectable in the lymph nodes, and that there is more pigment deposition and hyperplasia specifically in inguinal and axillary lymph nodes. The carbon particles mobilize and migrate through the lymphatics and can be seen within the macrophages or extracellularly. This migration is similar to that seen with melanoma cells. Melanoma metastasis was presumed in discolored, enlarged lymph nodes that

Magee-Womens Hospital of UPMC, Pittsburgh, PA, USA

Address for correspondence: A. Soran, MD, MPH, Magee-Womens Hospital, 300 Halket St Suite 2601, Pittsburgh, PA, 15213, U.S.A. Fax: +412.6411446

Acknowledgement: All of the authors declare that they have no conflict of interest in the information contained in the manuscript.

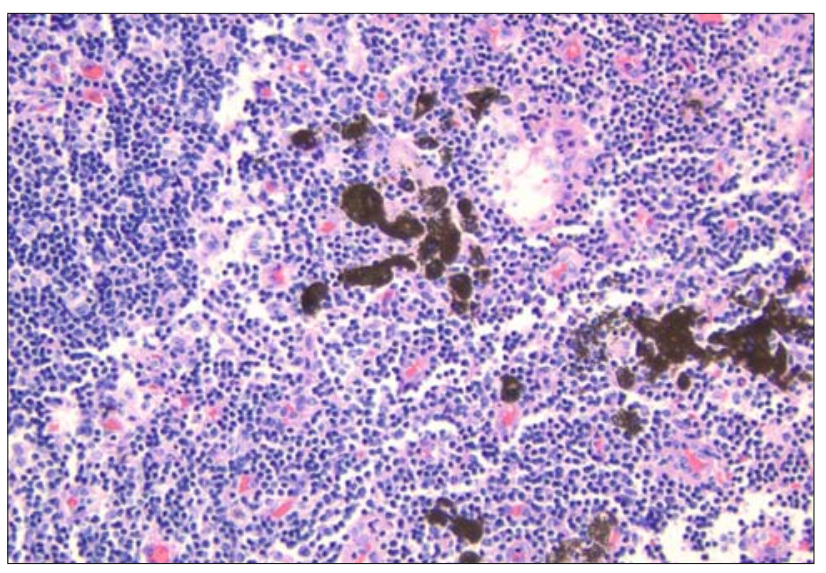

Fig. 1. Tattoo pigments deposits and within macrophages in Axillary Sentinel Lymph node (H\&E 20X).

were later found to have only extracellular tattoo pigment deposition and hyperplasia.

Additionally, the size of the tattoo appears independent of the amount of lymph node pigmentation. Even if the patient has removed the tattoo by laser surgery or dermabrasion, the lymph node pigmentation does not disappear (4-5). Here, we present a case report of a breast cancer patient with a tattoo and discuss the importance of tattoo pigment in the LN.

\section{Case report}

A 73-years-old female presented with complaint of right nipple bloody discharge. Physical exam revealed that the right nipple was inverted with no palpable lump masses. A tattoo on her shoulder and right chest wall was noted. The patient's mammogram showed 2 areas of calcifications, and subsequent core biopsy revealed ductal carcinoma in situ. Patient underwent right total mastectomy and sentinel lymph node biopsy. Intra-operatively four colored nodes were harvested, labeled and sent separately for histopatho- 
logy. The first and third nodes were hot with technetium 99 and contained blue dye staining, the second node was blue in color and the fourth axillary SLN was palpable only. The pathology report of the lymph nodes revealed that all (1st, 2nd, 3rd and 4th) axillary SLN were free of tumor. In addition, the second axillary SLN which was grossly blue/black in color had extracellular anthracotic pigmentation and pigment-laden macrophages (Fig. 1).

\section{Discussion}

Axillary lymph node involvement continues to be the most powerful prognostic predictor of breast cancer. Over the last decade, the axillary SLNB has replaced routine ALND for clinical staging in early breast cancer. In the SLN procedure, the use of both vital blue dyes and radiocolloid results in the highest rate of sentinel node identification. The optimal method of SLN identification remains controversial. SLN detection is more successful when using both periareolar intradermal injection of Tc-99m sulfur colloid and subareolar injection of blue dye techniques (6). Obtaining a careful tattoo history and physical examination are valuable in the evaluation of any patient undergoing work-up for breast cancer (7).

Studies describe a potential pitfall in the identification of a true sentinel node during surgery due to lymph node pigmentation secondary to migration of tattoo dye. These pigmented "pseudosentinel” nodes, if located superficially in the axilla, may mimic the blue sentinel node on visual inspection, therefore missing the true sentinel node and potentially understaging the patient. Only when these sentinel nodes are dissected and sent for pathological evaluation does the surgeon know if the node was a true SLN with blue dye and tumor deposits or a pseudo SLN with anthracotic pigment. These patients may need a second procedure at a later date for repeated axillary staging if this node is positive for tumor.

Tattoo pigmented lymph nodes may contain calcium and on mammography may give a false impression of being metastatic. Subsequent fine needle aspiration reveals black pigment and benign reactive lymph nodes. These pseudo sentinel nodes containing migrated carbonaceous particles from tattoos are also often enlarged secondary to reactive lymphoid hyperplasia, further giving the surgeon a false impression of a grossly metastatic lymph node. Upon finding an enlarged SLN stained grossly black and suspicious for metastatic disease, surgeons cannot determine whether the SLN represented a metastatic node for breast cancer or adenopathy due to pigmentation from tattoo. Rarely, when a SLN is suspicious based on palpation or overtly blue/black coloration, a complete lymph node dissection may be performed during the same operation without prior histological evaluation for metastasis. Surgeons should be aware of this phenomenon to avoid a complete lymph node dissection (8).

In conclusion, history taking and examination of a patient with breast cancer should include the presence of decorative tattoos especially in the drainage areas of the axilla. The surgeon should keep in mind that "pseudo-sentinel" nodes may mimic the true sentinel node on visual inspection, which may result in missing the true sentinel node and potentially understaging the breast cancer.

\section{References}

1. Heywood W, Patrick K, Smith A, et al. Who gets tattoos? Demographic and behavioral correlates of ever being tattooed in a representative sample of men and women. Ann Epidemiol 2012; 22: 51-56.

2. Hogsberg T, Carlsen H, Serup J. High prevalence of minor symptoms in tattoos among a young population tattooed with carbon black and organic pigments. J Eur Acad Dermatol Venereol 2012 doi: 10.1111/j.14683083.2012.04590.x

3. Statistic Brain. 2012. Pew Research Center, Tattoo Finder, Vanishing Tattoo. 23 July 2012. http://www.statisticbrain.com

4. Beavis A, Amneus M, Aoyama C, Holschneider C. Tattoo pigment lymphadenopathy mimicking metastasis in vulvar cancer. Obstet Gynecol 2012; 120: 442-444.

5. Chickkamuniyappa S, Sjuve-Scott R, Lancaster-Weiss K, Miller A, Yeh I. Tattoo pigment in sentinel lymph nodes: a mimicker of metastatic malignant melanoma. Dermatol Online J 2012; 11 (1).

6. Argon A, Duygun U, Acar $\mathbf{E}$ et al. The use of periareolar intradermal Tc-99m tin colloid and peritumoral intraparenchymal isosulfan blue dye injections for determination of the sentinel lymph node. Clin Nucl Med 2006; 31: 795-800.

7. Schlager A, Laser A, Melamed J, Guth A. A tattoo pigmented node masquerading as the sentinel node in a case of breast cancer. Breast $\mathbf{J}$ 2008; 14: 299-300.

8. Jakub J, Garcia E, Giuliano R, Pendas S, Reintgen D. A black SLN in melanoma patient. The Journal of Family Practice case report. http:// www.jfponline.com/pages.asp?aid=2063 